\title{
MINIMAX SOLUTIONS OF ORDINARY DIFFERENTIAL SYSTEMS
}

\author{
L. P. BURTON AND WILLIAM M. WHYBURN
}

1. Introduction. Let $f_{i}\left(x, y_{1}, \cdots, y_{n}\right), i=1, \cdots, n$, be defined and continuous over a region $R:\left(x, y_{1}, \cdots, y_{n}\right)$ of $(n+1)$-dimensional space. Let $P:\left(x_{0}, y_{10}, \cdots, y_{n 0}\right)$ be a point of $R$ and consider the differential system

$$
y_{i}^{\prime}=f_{i}\left(x, y_{1}, \cdots, y_{n}\right), \quad i=1, \cdots, n .
$$

The classical existence theorem of Peano states that there is at least one solution ${ }^{1} y_{1}(x), \cdots, y_{n}(x)$ of $(\mathrm{S})$ through $P$ existing in ${ }^{2} R$ over some interval $x_{0}-h \leqq x \leqq x_{0}+k$, where $h, k>0$. If for some such interval this solution through $P$ is not unique, then there are infinitely many solutions through $P$ and the existence of critical type solutions is a possibility. W. Osgood, ${ }^{3}$ P. Montel, ${ }^{4}$ and O. Perron, ${ }^{5}$ using different methods, considered the case $n=1$ and proved the existence of a maximum and a minimum solution. E. Kamke ${ }^{6}$ gave an example to show that for $n>1$ there will not in general be a maximum and a minimum solution through $P$, but that such solutions do exist provided $f_{i}\left(x, y_{1}, \cdots, y_{n}\right), i=1, \cdots, n$, satisfy certain monotone properties with respect to $y_{1}, \cdots, y_{n}$. This paper is devoted to establishing the existence of other types of critical solutions and to a consideration of some of their properties.

2. Notation. Hereafter whenever the subscript $i$ is used it is to be understood that $i$ ranges over the set $(1, \cdots, n)$. Similarly, the sub-

Presented to the Society, April 21, 1951; received by the editors August 1, 1951.

1 A solution of (S), as used throughout this paper, is by definition a set of differentiable functions $y_{1}(x), \cdots, y_{n}(x)$ for which $y_{i}^{\prime}(x)=f_{i}\left[x, y_{1}(x), \cdots, y_{n}(x)\right], i=1$, $\cdots, n$, identically over some interval $a \leqq x \leqq b$.

2 Here it is meant that each point of the curve in $(n+1)$-dimensional space given parametrically by $x=x, y_{1}=y_{1}(x), \cdots, y_{n}=y_{n}(x)$ lies in $R$ over $x_{0}-h \leqq x \leqq x_{0}+k$. For the sake of brevity this terminology will be used freely.

${ }^{3}$ Osgood, Beweis der Existenz einer Lösung der Differentialgleichung $d y / d x=f(x, y)$ ohne Hinzunahme der Cauchy-Lipschitz'schen Bedingung, Monatshefte für Mathematik und Physik vol. 9 (1898) pp. 331-345.

- Montel, Sur les suites infinies de fonctions, Ann. École Norm. vol. 24 (1907) pp. 233-234.

- Perron, Ein neuer Existenzbeweis für die Integrale der Differentialgleichung $y^{\prime}$ $=f(x, y)$, Math. Ann. vol. 76 (1915) pp. 471-484.

- Kamke, Zur Theorie der Systems gewöhnlicher Differentialgleichungen, Acta Math. vol. 58 (1932) pp. 57-85. 
scripts $p$ and $q$ will have the ranges $(1, \cdots, k)$ and $(k+1, \cdots, n)$, respectively.

For a fixed index $k, 1 \leqq k \leqq n$, a set of functions $f_{i}\left(x, y_{1}, \cdots, y_{n}\right)$ will be said to have property $\left(\pi_{k}\right)$ if, on the subset of $R$ for all of whose points $x \geqq x_{0}$, the following conditions are satisfied:

(1) $f_{i}$ is continuous in $\left(x, y_{1}, \cdots, y_{n}\right)$.

$\left(\pi_{k}\right) \quad$ monotone decreasing in $y_{q}$.

(2) $f_{p}$ is monotone increasing in $y_{j}, j=1, \cdots, k ; j \neq p$, and

(3) $f_{q}$ is monotone decreasing in $y_{p}$ and monotone increasing in $y_{j}, j=k+1, \cdots, n ; j \neq q$.

In a similar manner $\left(\pi_{\mathbf{k}}^{*}\right)$ will designate that set of conditions one obtains on the subset of $R$ for which $x \leqq x_{0}$ by interchanging the words "increasing" and "decreasing" in property $\left(\pi_{k}\right)$.

To shorten further the notation $h_{j}[x, y]$ will be used to represent $h_{j}\left(x, y_{1}, \cdots, y_{n}\right)$, and $W(x)$ will be used to represent the set of $n$ functions $w_{1}(x), w_{2}(x), \cdots, w_{n}(x)$. As in $\$ 1, P$ will designate a point $\left(x_{0}, y_{10}, \cdots, y_{n 0}\right)$ of a region $R$.

\section{Preliminary theorems.}

THEOREM 1. Let the following conditions hold over the subset of a region $R$ for all of whose points $x \geqq x_{0}$ :

(1) The functions $f_{i}[x, y]$ are continuous in $\left(x, y_{1}, \cdots, y_{n}\right)$.

(2) The set of functions $g_{i}[x, y]$ has property $\left(\pi_{k}\right)$.

(3) $f_{p}[x, y]<g_{p}[x, y], f_{q}[x, y]>g_{q}[x, y]$.

Let $Y(x)$ be a solution of $(S)$ through $P$ and $Z(x)$ be a solution of the system $y_{i}^{\prime}=g_{i}[x, y]$ through $P$. If both of these solutions exist in $R$ on $x_{0} \leqq x<x_{0}+a, a>0$, then on $x_{0}<x<x_{0}+a$

$$
y_{p}(x)<z_{p}(x) \text { and } y_{q}(x)>z_{q}(x) .
$$

Proof. Define $Q_{p}(x)=z_{p}(x)-y_{p}(x)$ and $Q_{q}(x)=y_{q}(x)-z_{q}(x)$. Then $Q_{i}\left(x_{0}\right)=0$ and by applying hypothesis (3) one sees that

$$
Q_{p}^{\prime}\left(x_{0}\right)=g_{p}\left[x_{0}, z\left(x_{0}\right)\right]-f_{p}\left[x_{0}, y\left(x_{0}\right)\right]>0
$$

and

$$
Q_{q}^{\prime}\left(x_{0}\right)=f_{q}\left[x_{0}, y\left(x_{0}\right)\right]-g_{q}\left[x_{0}, z\left(x_{0}\right)\right]>0 .
$$

Since $Q_{i}(x)$ is continuous on the right at $x_{0}$, it follows that $(\mathrm{H})$ is true over some interval $x_{0}<x<x_{0}+\delta, \delta>0$. Let $\gamma$ be the upper limit of the set of values $x_{0}+\delta$ for which $(\mathrm{H})$ is true on $x_{0}<x<x_{0}+\delta$. Assume that $\gamma$ is less than $x_{0}+a$. Then since $Q_{i}(x)$ is continuous and has a derivative on $x_{0} \leqq x<x_{0}+a$, we have for at least one $\mu$ 


$$
\begin{aligned}
Q_{\mu}(\gamma) & =0, \\
Q_{\mu}^{\prime}(\gamma) & \leqq 0, \\
Q_{i}(\gamma) & \geqq 0, \quad i \neq \mu .
\end{aligned}
$$

Relations (i) and (iii) give $z_{p}(\gamma) \geqq y_{p}(\gamma)$ and $z_{q}(\gamma) \leqq y_{q}(\gamma)$. By applying hypotheses (2) and (3) one obtains

(a) if $1 \leqq \mu \leqq k, f_{\mu}[\gamma, y(\gamma)]<g_{\mu}[\gamma, y(\gamma)] \leqq g_{\mu}[\gamma, z(\gamma)]$;

(b) if $k+1 \leqq \mu \leqq n, f_{\mu}[\gamma, y(\gamma)]>g_{\mu}[\gamma, y(\gamma)] \geqq g_{\mu}[\gamma, z(\gamma)]$.

From (ii) one obtains

(c) if $1 \leqq \mu \leqq k, z_{\mu}^{\prime}(\gamma) \leqq y_{\mu}^{\prime}(\gamma)$; i.e., $g_{\mu}[\gamma, z(\gamma)] \leqq f_{\mu}[\gamma, y(\gamma)]$;

(d) if $k+1 \leqq \mu \leqq n, y_{\mu}^{\prime}(\gamma) \leqq z_{\mu}^{\prime}(\gamma)$; i.e., $f_{\mu}[\gamma, y(\gamma)] \leqq g_{\mu}[\gamma, z(\gamma)]$.

Either (a) and (c) or (b) and (d) are contradictory. Hence $\gamma \geqq x_{0}+a$.

COROLLARy. The conclusion of Theorem 1 still follows if the conditions (1) and (2) are replaced by:

$\left(1^{\prime}\right)$ The functions $g_{i}[x, y]$ are continuous;

$\left(2^{\prime}\right)$ The set of functions $f_{i}[x, y]$ has property $\left(\pi_{k}\right)$.

Proof. Proceeding as before one obtains

$$
\begin{aligned}
& \text { if } 1 \leqq \mu \leqq k, f_{\mu}[\gamma, y(\gamma)] \leqq f_{\mu}[\gamma, z(\gamma)]<g_{\mu}[\gamma, z(\gamma)], \\
& \text { if } k+1 \leqq \mu \leqq n, f_{\mu}[\gamma, y(\gamma)] \geqq f_{\mu}[\gamma, z(\gamma)]>g_{\mu}[\gamma, z(\gamma)] .
\end{aligned}
$$

Thus the relations (a) and (b) still hold. The remainder of the proof is identical to the proof of the theorem.

THEOREM 2. Let the following conditions hold over the subset of $R$ for all of whose points $x \leqq x_{0}$ :

(1) The functions $f_{i}[x, y]$ are continuous in $\left(x, y_{1}, \cdots, y_{n}\right)$.

(2) The set of functions $g_{i}[x, y]$ has property $\left(\pi_{i}^{*}\right)$.

(3) $f_{p}[x, y]>g_{p}[x, y], f_{q}[x, y]<g_{q}[x, y]$.

Let $Y(x)$ be a solution of $(\mathrm{S})$ through $P$ and $Z(x)$ be a solution of the system $y_{i}^{\prime}=g_{i}[x, y]$ through $P$. If both of these solutions exist in $R$ on $x_{0}-a<x \leqq x_{0}, a>0$, then on $x_{0}-a<x<x_{0}$

$$
y_{p}(x)<z_{p}(x) \text { and } y_{q}(x)>z_{q}(x) .
$$

The proof of this theorem is analogous to the proof of Theorem 1. Obviously this theorem has a corollary similar to that of Theorem 1 .

4. Definition of minimax solutions.

Definition. Let $\Phi(x)$ be a solution of (S) through $P$ existing over an interval $I: x_{0}-h_{1}<x<x_{0}+h_{2}\left(h_{1}, h_{2} \geqq 0, h_{1}+h_{2}>0\right)$ such that for any 
other solution $Y(x)$ of $(\mathrm{S})$ through $P$ the relations

$$
y_{p}(x) \leqq \phi_{p}(x), \quad y_{q}(x) \geqq \phi_{q}(x)
$$

or

$$
y_{p}(x) \geqq \phi_{p}(x), \quad y_{q}(x) \leqq \phi_{q}(x)
$$

hold over any subinterval of $I$ where $Y(x)$ exists. In case (A) the solution $\Phi(x)$ is designated as a (k)max- $(n-k)$ min solution of $(\mathrm{S})$ through $P$ over I and in case (B) as a (k) min- $(n-k)$ max solution of (S) through $P$ over $I$. In either case it is called a minimax solution.

If $Y(x)$ is a $(k) \max -(n-k)$ min solution of (S) through $P$, it will be indicated as $U_{1}(x), \cdots, U_{k}(x), u_{k+1}(x), \cdots, u_{n}(x)$. Similarly $u_{1}(x), \cdots, u_{k}(x), U_{k+1}(x), \cdots, U_{n}(x)$ will designate a $(k)$ min$(n-k)$ max solution.

5. Existence of minimax solutions. Consider the systems

$$
\begin{aligned}
& y_{p}^{\prime}=f_{p}\left(x, y_{1}, \cdots, y_{n}\right)+\epsilon, \\
& y_{q}^{\prime}=f_{q}\left(x, y_{1}, \cdots, y_{n}\right)-\epsilon
\end{aligned}
$$

Hereafter the symbol $Y(x, \epsilon)$ will be used to designate the set of $n$ functions $y_{1}(x, \epsilon), \cdots, y_{n}(x, \epsilon)$.

Lemma 1. Let the functions $f_{i}[x, y]$ be continuous in $R$. Then there exists an $\epsilon_{0}>0$ and an $r>0$ such that all solutions of $(\mathrm{S})$ and $\left(\mathrm{S}_{\mathrm{\epsilon}}\right)$, for $0<\epsilon<\epsilon_{0}$, through $P$, exist in $R$ on the interval $\left|x-x_{0}\right| \leqq r$.

Proof. Take any hyper-rectangle $B:\left|x-x_{0}\right| \leqq a,\left|y_{i}-y_{i 0}\right| \leqq b$ lying in $R$. Let $Y(x)$ and $Y(x, \epsilon)$ be arbitrary solutions of $(\mathrm{S})$ and $\left(\mathrm{S}_{\epsilon}\right)$, respectively, through $P$. These solutions exist ${ }^{7}$ to the boundary of $R$. Let $M$ be the maximum value in $B$ of $\left|f_{i}[x, y]\right|$. Take $\epsilon_{0}=M$ and $r=\min (a, b / 2 M)$. For any $x$ such that $\left|x-x_{0}\right| \leqq r$ and any $\epsilon$ such that $0<\epsilon<\epsilon_{0}$

$$
\begin{aligned}
\left|y_{i}(x, \epsilon)-y_{i 0}\right| & =\left|\int_{x_{0}}^{x}\left\{f_{i}[t, y(t, \epsilon)] \pm \epsilon\right\} d t\right| \\
& \leqq M\left|x-x_{0}\right|+\epsilon_{0}\left|x-x_{0}\right| \leqq 2 M r \leqq b
\end{aligned}
$$

and

$$
\left|y_{i}(x)-y_{i 0}\right|=\left|\int_{x_{0}}^{x} f_{i}[t, y(t)] d t\right| \leqq M r \leqq \frac{b}{2}
$$

'Cf., for example, Kamke, Differentialgleichungen reeller Funktionen, Leipzig, 1930, p. 135. 
THEOREM 3. Let the set of functions $f_{i}[x, y]$ have property $\left(\pi_{k}\right)$. Then the system (S) has a unique $(k) \max -(n-k) \min$ solution $U_{1}(x), \cdots$, $U_{k}(x), u_{k+1}(x), \cdots, u_{n}(x)$, and a unique $(k)$ min- $(n-k)$ max solution $u_{1}(x), \cdots, u_{k}(x), U_{k+1}(x), \cdots, U_{n}(x)$ through $P$. These solutions exist at least on an interval $x_{0} \leqq x<x_{0}+r, r>0$.

Proof. Use the notation of Lemma 1 so that on the interval $I_{r}: x_{0}-r \leqq x \leqq x_{0}+r$ any solution $Y(x)$ of $(\mathrm{S})$ and $Y(x, \epsilon)$ of $\left(\mathrm{S}_{\epsilon}\right)$ $\left(0<\epsilon<\epsilon_{0}=M\right)$ through $P$ exists in $R$. Let $j$ be any fixed index such that $1 \leqq j \leqq n$. The set of functions $\left\{y_{j}(x, \epsilon)\right\}$ is uniformly bounded and equicontinuous on $I_{r}$. For

$$
\begin{aligned}
y_{j}(x, \epsilon) & =y_{j 0}+\int_{x_{0}}^{x} f_{j}[t, y(t, \epsilon)] d t \pm\left(x-x_{0}\right) \epsilon, \\
\left|y_{j}(x, \epsilon)\right| & \leqq\left|y_{j 0}\right|+\left(M+\epsilon_{0}\right) r=\left|y_{j 0}\right|+2 M r
\end{aligned}
$$

and

$$
\begin{aligned}
\left|y_{j}\left(x_{2}, \epsilon\right)-y_{j}\left(x_{1}, \epsilon\right)\right|= & \mid \int_{x_{0}}^{x_{2}} f_{j}[t, y(t, \epsilon)] d t \pm\left(x_{2}-x_{0}\right) \epsilon \\
& -\int_{x_{0}}^{x_{1}} f_{j}[t, y(t, \epsilon)] d t \mp\left(x_{1}-x_{0}\right) \epsilon \mid \\
& =\left|\int_{x_{1}}^{x_{2}} f_{j}[t, y(t, \epsilon)] d t \pm\left(x_{2}-x_{1}\right) \epsilon\right| \\
& \leqq 2 M\left|x_{2}-x_{1}\right| .
\end{aligned}
$$

If $\epsilon_{1}$ and $\epsilon_{2}$ are chosen so that $0<\epsilon_{2}<\epsilon_{1}<\epsilon_{0}$, then

$$
f_{p}[x, y]+\epsilon_{1}>f_{p}[x, y]+\epsilon_{2}, \text { and } f_{q}[x, y]-\epsilon_{1}<f_{q}[x, y]-\epsilon_{2} \text {. }
$$

A straightforward application of Theorem 1 gives

$$
y_{p}\left(x, \epsilon_{1}\right) \geqq y_{p}\left(x, \epsilon_{2}\right) \text { and } y_{q}\left(x, \epsilon_{1}\right) \leqq y_{q}\left(x, \epsilon_{2}\right)
$$

over $x_{0} \leqq x \leqq x_{0}+r$. Hence over this interval the functions $y_{j}(x, \epsilon)$ are monotone increasing functions of $\epsilon$ if $j=1, \cdots, k$, and monotone decreasing functions of $\epsilon$ if $j=k+1, \cdots, n$. Choose a sequence $\left\{\epsilon_{m}\right\}$ such that $\epsilon_{0}>\epsilon_{1}>\epsilon_{2} \cdots$ and $\lim _{m \rightarrow \infty} \epsilon_{m}=0$. By the use of Ascoli's Theorem together with the monotone properties of the functions $y_{j}\left(x, \epsilon_{m}\right)$ one establishes the existence of a limiting function $y_{j}^{*}(x)$ to which the sequence $\left\{y_{j}\left(x, \epsilon_{m}\right)\right\}$ converges uniformly over $x_{0} \leqq x$ $\leqq x_{0}+r$. By applying Theorem 1 again it is clear that $\lim _{\epsilon \rightarrow 0} y_{i}(x, \epsilon)$ $=y_{i}^{*}(x)$ and the convergence is uniform over $x_{0} \leqq x \leqq x_{0}+r$.

The set of functions $y_{1}^{*}(x), \cdots, y_{n}^{*}(x)$ is a solution of $(\mathrm{S})$ on 
$x_{0} \leqq x \leqq x_{0}+r$. For

$$
\begin{aligned}
y_{i}^{*}(x)=\lim _{\epsilon \rightarrow 0} y_{i}(x, \epsilon) & =\lim _{\bullet \rightarrow 0}\left\{y_{i 0}+\int_{x_{0}}^{x} f_{i}[t, y(t, \epsilon)] d t \pm\left(x-x_{0}\right) \epsilon\right\} \\
& =y_{i 0}+\int_{x_{0}}^{x} \lim _{\leftrightarrow \rightarrow 0} f_{i}[t, y(t, \epsilon)] d t
\end{aligned}
$$

since $f_{i}[t, y(t, \epsilon)]$ converges uniformly to $f_{i}\left[t, y^{*}(t)\right]$ on $x_{0} \leqq x \leqq x_{0}+r$. Then $y_{i}^{*}(x)=y_{i 0}+\int_{x_{0}}^{x} f_{i}\left[t, y^{*}(t)\right] d t$, by the continuity of the functions $f_{i}[x, y]$.

Now consider an arbitrary solution $Y(x)$ of $(\mathrm{S})$ through $P$. Notice that

$$
f_{p}[x, y]<f_{p}[x, y]+\epsilon \text { and } f_{q}[x, y]>f_{q}[x, y]-\epsilon
$$

and apply Theorem 1 . One finds that over $x_{0} \leqq x \leqq x_{0}+r$

$$
y_{p}(x) \leqq y_{p}(x, \epsilon) \text { and } y_{q}(x) \geqq y_{q}(x, \epsilon) .
$$

Consequently

$$
v_{p}(x) \leqq y_{p}^{*}(x)=\lim _{\epsilon \rightarrow 0} y_{p}(x, \epsilon) \text { and } y_{q}(x) \geqq y_{q}^{*}(x)=\lim _{\epsilon \rightarrow 0} y_{q}(x, \epsilon) .
$$

This proves that on $x_{0} \leqq x<x_{0}+r$

$$
y_{p}^{*}(x)=U_{p}(x) \text { and } y_{q}^{*}(x)=u_{q}(x) .
$$

The uniqueness of the solution $U_{1}(x), \cdots, U_{k}(x), u_{k+1}(x), \cdots, u_{n}(x)$ follows immediately from its definition.

The proof of the existence of a unique solution $u_{1}(x), \cdots, u_{k}(x)$, $U_{k+1}(x), \cdots, U_{n}(x)$ is made in a similar manner by considering, instead of the system $\left(\mathrm{S}_{\boldsymbol{\varepsilon}}\right)$, the system

$$
y_{p}^{\prime}=f_{p}[x, y]-\epsilon, \quad y_{q}^{\prime}=f_{q}[x, y]+\epsilon .
$$

THEOREM 4. Let the set of functions $f_{i}[x, y]$ have property $\left(\pi_{k}^{*}\right)$. Then the system (S) has a unique $(k) \max -(n-k) \min$ solution, $U_{1}(x), \cdots, U_{k}(x), u_{k+1}(x), \cdots, u_{n}(x)$, and a unique $(k)$ min$(n-k)$ max solution $u_{1}(x), \cdots, u_{k}(x), U_{k+1}(x), \cdots, U_{n}(x)$, through $P$. These solutions exist at least over an interval $x_{0}-r<x \leqq x_{0}(r>0)$.

The proof of this theorem is analogous to that of Theorem 3 .

EXAmple. Consider the system $y_{1}^{\prime}=-5 x y_{2}, y_{2}^{\prime}=-3 x\left(y_{1}\right)^{1 / 5}$. Let $R$ be any region containing the point $P:(0,0)$. It is immediately seen that the functions $f_{1}\left(x, y_{1}, y_{2}\right) \equiv-5 x y_{2}$ and $f_{2}\left(x, y_{1}, y_{2}\right) \equiv-3 x\left(y_{1}\right)^{1 / 5}$ have properties $\left(\pi_{1}\right)$ and $\left(\pi_{1}^{*}\right)$ in $R$. For $x \geqq 0$ the given system has the solutions $U_{1}(x)=x^{5}, u_{2}(x)=-x^{3}$ and $u_{1}(x)=0, U_{2}(x)=0$. For $x \leqq 0$ 
it has the solutions $U_{1}(x)=-x^{3}, u_{2}(x)=x^{5}$ and $u_{1}(x)=0, U_{2}(x)=0$.

6. Extension of minimax solutions to the boundary $R$. The notation $C_{G}$ will be used to represent the curve in $(n+1)$-dimensional space determined by the equations $x=x, y_{i}=g_{i}(x)$. Similarly $C_{G \mathrm{c}}$ will be used to represent the curve determined by equations of the type $x=x, y_{i}=g_{i}(x, \epsilon)$. A curve of either type will be said to lie in $R$ over an interval if for each value of $x$ in this interval the corresponding point of the curve is in $R$.

We know from the proof of Theorem 3 that there exists some interval $x_{0} \leqq x \leqq x_{0}+r$ on which the functions $y_{j}(x, \epsilon)$ converge uniformly to a function $y_{j}^{*}(x)$ such that $C_{Y *}$ lies in $R$.

LEMMA 2. Let the set of functions $f_{i}[x, y]$ have property $\left(\pi_{k}\right)$. Let $Y(x, \epsilon)$ be a solution of $\left(\mathrm{S}_{\epsilon}\right)$ through $P$, and $X:\left(x_{0}, b\right), b>x_{0}$, be the greatest interval to the right of $x_{0}$ over which, for all fixed indices $j$, $1 \leqq j \leqq n$, the functions $y_{j}(x, \epsilon)$ converge uniformly (as $\epsilon$ approaches zero) to a function $y_{j}^{*}(x)$ so that $C_{Y}$. lies in $R$. Then the point $\left[b, y_{1}^{*}(b), \cdots, y_{n}^{*}(b)\right]$ lies in the boundary of $R$.

Proof. It will first be proved that $X$ cannot be a closed interval. If so, $C_{Y} \cdot$ exists in $R$ over $x_{0} \leqq x \leqq b$ and $\left[b, y_{1}^{*}(b), \cdots, y_{n}{ }^{*}(b)\right]$ lies in $R$. Choose a hyper-rectangle $B:|x-b| \leqq \alpha,\left|y_{i}-y_{i}^{*}(b)\right| \leqq \beta(0<\alpha<b$ $\left.-x_{0}, \beta>0\right)$ lying in $R$. By the property of uniform convergence there exists an $\epsilon_{1}>0$ such that for $\epsilon<\epsilon_{1}$ the curves $C_{Y_{\epsilon}}$ lie in $R$ and also

$$
\left|y_{i}(x, \epsilon)-y_{i}^{*}(x)\right|<\beta / 2 \text { on } x_{0} \leqq x \leqq b .
$$

The curves $C_{Y_{\epsilon}}$ are known to exist to the boundary of $R$. Now let $M=\max \left\{\left|f_{i}\left(x, y_{1}, \cdots, y_{n}\right)\right|+\left|\epsilon_{1}\right|\right\}$ in $B$. Then if $\epsilon<\epsilon_{1}, C_{Y_{e}}$ exists in $B$ over $b \leqq x \leqq b+\beta / 2 M$. For over this interval

$$
\begin{aligned}
\left|y_{i}(x, \epsilon)-y_{i}^{*}(b)\right| & \leqq\left|y_{i}(x, \epsilon)-y_{i}(b, \epsilon)\right|+\left|y_{i}(b, \epsilon)-y_{i}^{*}(b)\right| \\
& \leqq M|x-b|+\beta / 2 \leqq \beta .
\end{aligned}
$$

Thus the curves $C_{Y_{\epsilon}}, \epsilon<\epsilon_{1}$, exist in $R$ over the interval $x_{0} \leqq x \leqq b$ $+\beta / 2 M$. It can be shown as in the proof of Theorem 3 that over this interval, for a fixed $j$, the set $\left\{y_{j}(x, \epsilon)\right\}$ is uniformly bounded and equicontinuous and monotone increasing in $\epsilon$ if $j=1, \cdots, k$, monotone decreasing in $\epsilon$ if $j=k+1, \cdots, n$. Consequently $\lim _{\epsilon \rightarrow 0} y_{j}(x, \epsilon)$ exists uniformly over $\left(x_{0}, x_{0}+b+\beta / 2 M\right)$. This contradicts the definition of $X$.

Now assume that $X$ is the interval $x_{0} \leqq x<b$; i.e., $X$ is open on the right. If $\left[b, y_{1}^{*}(b), \cdots, y_{n}^{*}(b)\right]$ lies in $R$, where by definition $y_{i}^{*}(b)$ $=\lim _{x \rightarrow b^{-}} y_{i}^{*}(x)$, then $\lim _{\epsilon \rightarrow 0} y_{i}(x, \epsilon)=y_{i}^{*}(x)$ uniformly over the closed 
interval $x_{0} \leqq x \leqq b$. For, if $\epsilon$ is sufficiently small, we have for an arbitrary $\eta>0$

$$
\left|y_{i}(x, \epsilon)-y_{i}^{*}(x)\right|<\eta / 2<\eta \text { on } x_{0} \leqq x<b .
$$

Consequently,

$$
\left|y_{i}(b, \epsilon)-y_{i}^{*}(b)\right| \leqq \eta / 2<\eta
$$

and

$$
\left|y_{i}(x, \epsilon)-y_{i}^{*}(x)\right|<\eta \text { on } x_{0} \leqq x \leqq b .
$$

This again contradicts the fact that $X$ is by definition the greatest interval such that $C_{Y}$ lies in $R$. Hence the point $\left[b, y_{1}^{*}(b), \cdots, y_{n}^{*}(b)\right\}$ lies in the boundary of $R$.

An argument entirely similar to that used in the proof of Theorem 3 proves that the set of functions $y_{1}^{*}(x), \cdots, y_{n}^{*}(x)$ constitutes a solution of $(\mathrm{S})$ over $x_{0} \leqq x<b$. Now let $Y(x)$ be an arbitrary solution of (S) through $P$ existing in $R$ on $x_{0} \leqq x<b<b$. If $\epsilon$ is taken sufficiently small to insure the existence in $R$ of $C_{Y_{\epsilon}}$ on $x_{0} \leqq x \leqq b$, an application of Theorem 1 shows that over this interval

$$
y_{p}(x) \leqq y_{p}(x, \epsilon) \text { and } y_{q}(x) \geqq y_{q}(x, \epsilon) \text {, }
$$

and hence $y_{p}(x) \leqq y_{p}^{*}(x)$ and $y_{q}(x) \geqq y_{q}^{*}(x)$. This argument together with an obvious modification based on the system

$$
y_{p}^{\prime}=f_{p}[x, y]-\epsilon, \quad y_{q}^{\prime}=f_{q}[x, y]+\epsilon
$$

proves the following:

THEOREM 5. Let the set of functions $f_{i}[x, y]$ have property $\left(\pi_{k}\right)$. Then the solutions $U_{1}(x), \cdots, U_{k}(x), u_{k+1}(x), \cdots, u_{n}(x)$ and $u_{1}(x), \cdots$, $u_{k}(x), U_{k+1}(x), \cdots, U_{n}(x)$ of $(\mathrm{S})$ through $P$ exist for $x \geqq x_{0}$ to the boundary of $R$.

Analogous to Theorem 5 we have:

THEOREM 6. Let the set of functions $f_{i}[x, y]$ have property $\left(\pi_{k}^{*}\right)$. Then the solutions $U_{1}(x), \cdots, U_{k}(x), u_{k+1}(x), \cdots, u_{n}(x)$ and $u_{1}(x), \cdots$, $u_{k}(x), U_{k+1}(x), \cdots, U_{n}(x)$ of $(\mathrm{S})$ through $P$ exist for $x \leqq x_{0}$ to the boundary of $R$.

7. Approximation of minimax solutions by sets of under-over functions.

Definition. ${ }^{8}$ With reference to (S) and $P$ a set of (k)over-

8 The sets of under-over functions defined here are generalizations of the under and over functions originated by $\mathrm{O}$. Perron, loc. cit. 
$(n-k)$ under functions is a set of $n$ functions $\phi_{1}(x), \cdots, \phi_{n}(x)$ which are finite and continuous over an interval $x_{0} \leqq x \leqq x_{0}+a, a>0$, which have left-and right-hand derivatives over this interval, such that

$\phi_{i}\left(x_{0}\right)=y_{i 0}, \quad D_{ \pm} \phi_{p}(x)>f_{p}[x, \phi(x)] \quad$ and $\quad D_{ \pm} \phi_{q}(x)<f_{q}[x, \phi(x)]$.

Similarly $\phi_{1}(x), \cdots, \phi_{n}(x)$ constitutes a set of $(k)$ under- $(n-k)$ over functions if the above conditions hold except that

$$
D_{ \pm} \phi_{p}(x)<f_{p}[x, \phi(x)] \text { and } D_{ \pm} \phi_{q}(x)>f_{q}[x, \phi(x)] .
$$

TheOREM 7. Let the functions $f_{i}[x, y]$ have property $\left(\pi_{k}\right)$. Let $I: x_{0}$ $\leqq x \leqq x_{0}+a, a>0$, be an interval over which the minimax solutions of (S) through $P$ determine curves which lie in $R$. Then the solution $U_{1}(x), \cdots, U_{k}(x), u_{k+1}(x), \cdots, u_{n}(x)$ can be uniformly approximated over $I$ by $a$ set of $(k)$ over- $(n-k)$ under functions. Similarly the solution $u_{1}(x), \cdots, u_{k}(x), U_{k+1}(x), \cdots, U_{n}(x)$ can be uniformly approximated over I by $a$ set of $(k)$ under- $(n-k)$ over functions. ${ }^{9}$

Proof. A solution of $\left(\mathrm{S}_{\boldsymbol{6}}\right)$ through $P$ constitutes a set of $(k)$ over$(n-k)$ under functions. A solution of $\left(\mathrm{S}_{\epsilon}^{\prime}\right)$ through $P$ constitutes a set of $(k)$ under- $(n-k)$ over functions. It has already been shown (in the proofs of Theorem 3 and Lemma 2) that over $I$ solutions of $\left(S_{\epsilon}\right)$ and $\left(S_{\epsilon}^{\prime}\right)$ uniformly approach the minimax solutions as limiting functions.

8. Other properties of minimax solutions.

Definition. Assume that all solutions of $(\mathrm{S})$ through $P$ exist in $R$ over $I: x_{0}-h<x<x_{0}+k(h \geqq 0, k \geqq 0)$. The tube of solutions; $T_{P}$ of $(\mathrm{S})$ through $P$ over $I$ is the set of all points $\left[x, y_{1}(x), \cdots, y_{n}(x)\right]$ in $(n+1)$-dimensional space belonging to any solution through $P$ and having an abscissa $x$ such that $x_{0}-h<x<x_{0}+k$.

Let $T_{P}$ exist in $R$ on an interval $I: a<x<b$. Due to the strict inequality of relations $(\mathrm{H})$ and $\left(\mathrm{H}^{\prime}\right)$ of Theorems 1 and 2, respectively, we know that for an arbitrary solution $Y(x)$ of $(\mathbf{S})$ and $Y(x, \epsilon)$ of $\left(\mathbf{S}_{\epsilon}\right)$

$$
y_{i}(x)<y_{i}(x, \epsilon) \text { or } y_{i}(x)>y_{i}(x, \epsilon)
$$

for $x$ in $I, x \neq x_{0}, \epsilon>0$. Thus no point of any curve $C_{Y_{e}}$, having an abscissa $x \neq x_{0}$, is in $T_{P}$. But for $\epsilon$ sufficiently small, points of such curves are arbitrarily near any point of a curve lying in $T_{P}$ and determined by a minimax solution. This proves:

THEOREM 8. Under the hypothesis $\left(\pi_{k}\right)$ and/or the hypothesis $\left(\pi_{k}^{*}\right)$

9 The definitions of sets of under-over functions can be modified so that a similar theorem can be stated with respect to an interval $x_{0}-a \leqq x \leqq x_{0}, a>0$. 
the curve in $R$ determined by a minimax solution of (S) through $P$ lies entirely in the boundary of $T_{P}$.

Now let $T_{P}(b)$ be the intersection of $T_{P}$ with the hyperplane $x=b$. $T_{P}(b)$ is a continuum by a theorem of $\mathrm{H}$. Kneser. ${ }^{10}$

Theorem 9. If $j$ is any fixed index, $1 \leqq j \leqq n$, and $B_{j}$ is a number such that

$$
\text { g.l.b. }\left\{y_{j}(b)\right\}<B_{j}<\text { l.u.b. }\left\{y_{j}(b)\right\} \text {, }
$$

where $\left\{y_{j}(b)\right\}$ is the set of all $y_{j}(b)$ values in $T_{P}(b)$, then there exists $a$ solution of $(\mathrm{S})$ such that $y_{j}(b)=B_{j}{ }^{11}$

Proof. Otherwise the intersection of the hyperplanes $x=b, y_{j}=b_{j}$ divides the continuum $T_{P}(b)$ into two disjoint, nonempty sets which have no limit point in common.

COROLlaRY 1. If the set of functions $f_{i}[x, y]$ satisfies the hypothesis $\left(\pi_{k}\right)$ or $\left(\pi_{k}^{*}\right), B_{j}$ is a number such that $u_{j}(b) \leqq B_{j} \leqq U_{j}(b)$.

COROLlary 2. There exists a solution of (S) satisfying the $n+1$ boundary conditions $y_{i}\left(x_{0}\right)=y_{i 0}, y_{j}(b)=B_{j}$.

Finally, one may observe that the results of this paper, together with those of the papers cited, exhibit a strong similarity between critical point theory for functions of several real variables and critical solution theory for systems of ordinary differential equations. It is believed that further comparison of the two theories will yield other striking analogies.

The University of California, Davis and The University of North Carolina

10 Preuss. Akad. Wiss. Sitzungsber. Phys.-Math. Klasse, 1923. Another proof has been given by M. Müller, Math. Zeit. vol. 28 (1928) pp. 349-355.

11 This is a generalization of a theorem of G. Mie which can be stated as follows: Let $f(x, y)$ be continuous over a region $R$ of the $x y$-plane and $P:\left(x_{0}, y_{0}\right)$ be a point of $R$. Let $u(x)$ be the minimum solution through $P$ and $U(x)$ be the maximum solution through $P$ of the equation $y^{\prime}=f(x, y)$. Let $u(x)$ and $U(x)$ exist in $R$ over the interval $x_{0}-h<x<x_{0}+k$, where $h \geqq 0, k \geqq 0$. Then if $Q:(\xi, \eta)$ is a point such that $x_{0}-h<\xi<x_{0}+k, u(\eta) \leqq \eta \leqq U(\eta)$, there exists at least one solution of $y^{\prime}=f(x, y)$ through $P$ and $Q$. 